\title{
Correction: Radiotherapy increases plasma levels of tumoral cell-free DNA in non-small cell lung cancer patients
}

\author{
Shun-Ichiro Kageyama1,3, Keiji Nihei ${ }^{1}$, Katsuyuki Karasawa ${ }^{1}$, Takeshi Sawada ${ }^{1}$, \\ Fumiaki Koizumi ${ }^{1}$, Shigeo Yamaguchi ${ }^{2}$, Shunsuke Kato ${ }^{2}$, Hidehiro Hojo ${ }^{3}$, Atsushi \\ Motegi $^{3}$, Katsuya Tsuchihara ${ }^{3}$ and Tetsuo Akimoto ${ }^{3}$ \\ ${ }^{1}$ Tokyo Metropolitan Cancer and Infectious Diseases Center Komagome Hospital, Bunkyo-ku, Tokyo 113-8677, Japan \\ 2 Juntendo University Main Hospital, Tokyo 113-8431, Japan \\ ${ }^{3}$ National Cancer Center Hospital East, Kashiwa, Chiba 277-8577, Japan \\ Published: May 04, 2018
}

Copyright: Kageyama et al. This is an open-access article distributed under the terms of the Creative Commons Attribution License 3.0 (CC BY 3.0), which permits unrestricted use, distribution, and reproduction in any medium, provided the original author and source are credited.

This article has been corrected: The correct author names and affiliations are given below:

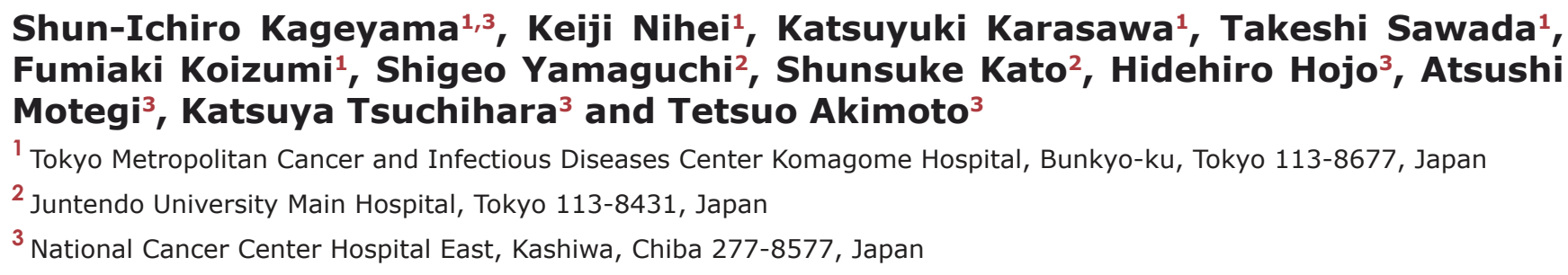
Fumiaki Koizumi ${ }^{1}$, Shigeo Yamaguchi ${ }^{2}$, Shunsuke Kato ${ }^{2}$, Hidehiro Hojo ${ }^{3}$, Atsushi Motegi $^{3}$, Katsuya Tsuchihara ${ }^{3}$ and Tetsuo Akimoto ${ }^{3}$

${ }^{1}$ Tokyo Metropolitan Cancer and Infectious Diseases Center Komagome Hospital, Bunkyo-ku, Tokyo 113-8677, Japan

2 Juntendo University Main Hospital, Tokyo 113-8431, Japan

${ }^{3}$ National Cancer Center Hospital East, Kashiwa, Chiba 277-8577, Japan

Original article: Oncotarget. 2018; 9:19368-19378. https://doi.org/10.18632/oncotarget.25053 